\title{
Hantavirus pulmonary syndrome: when should you consider this diagnosis?
}

Authors

Marcelo Carneiro ${ }^{1}$

Bruna Elisa Koch ${ }^{2}$

Eliane C Krummenauer ${ }^{3}$

Janete Aparecida

Machado ${ }^{4}$

${ }^{1} \mathrm{MSc}$ in Microbiology, Universidade Estadual de Londrina (UEL), PR; Specialist in Infectology, UEL, PR; Professor of Infectology, Universidade de Santa Cruz do Sul (UNISC), RS; Coordinator, Epidemiology Unit, Hospital Santa Cruz (HSC), RS, Brazil

${ }^{2}$ Medical Student, UNISC, RS; Trainee at CCIH HSC, RS, Brazil ${ }^{3}$ Specialist, ViceCoordinator,

Epidemiology Unit, HSC, RS, Brazil

${ }^{4}$ Specialist, Technician, Epidemiology Unit, HSC, RS, Brazil
Submitted on: 02/06/2011 Approved on: 02/23/2011

Correspondence to: Marcelo Carneiro Rua Thomaz Flores, $887 / 301$

Santa Cruz do Sul - RS

96810-090

carneiromarcelo@

yahoo.com.br

We declare no conflict of interest.
Dear Editor,

We describe two cases of Hantavirus infection in the town of Santa Cruz do Sul, state of Rio Grande do Sul, Brazil, that mimicked primarily community-acquired pneumonia.

Case 1 was a 28 -year-old married male individual, who worked as a farmer. On 11/11/09, he came to the Emergency Ward, with a history of fever, dyspnea, general myalgia (predominantly in the calves), nausea, tachycardia, periods of hypotension, weight loss and night sweats in the past three days. A chest $\mathrm{x}$-ray showed nonspecific interstitial infiltrate. The patient was admitted to the Intensive Care Unit (ICU) due to hemodynamic and respiratory instability for noninvasive ventilatory support, without vasoactive drugs. Empiric antimicrobial therapy was started with levofloxacin and sulfamethoxazole/trimethoprim, due to the diagnostic hypotheses of severe community-acquired pneumonia and pneumocystosis related to possible acquired immunodeficiency, which was later ruled out (nonreactive anti-HIV test); treatment was withdrawn and the patient was maintained on quinolone therapy, being discharged from the ICU on 11/12/09 and from the hospital on 11/17/09.

Case 2 was a 15-year-old high-school female student. On 11/30/09, she started to have fever, disseminated macular exanthema that progressed into petechiae, mainly in the lower limbs, and periods of hypotension. On $12 / 01 / 09$, a chest $\mathrm{x}$-ray disclosed diffuse bilateral infiltrate and a chest tomography confirmed the findings, showing ground-glass opacities in the central pulmonary areas with lymphatic thickening and moderate bilateral pleural effusion. After two days, the patient's clinical condition worsened (with somnolence, confusion, hemoptoic vomiting, tachypnea and dyspnea) and she was admitted to the ICU for respiratory monitoring and noninvasive mechanical ventilation, with vasoactive drugs.
Treatment was started with ceftriaxone and azithromycin due to the hypothesis of severe community-acquired pneumonia and meningococcemia (emergency diagnosis). On $12 / 08 / 2009$ the patient was discharged from the ICU, in spite of alterations in liver injury tests and was finally discharged from the hospital on 12/09/2009.

The Santa Cruz do Sul region is endemic for Leptospirosis and Hantaviruses in RS. Since 2006, the protocols of severe pneumonia cases include serology for these two types of hemorrhagic fevers, when relevant (in Case 1, thrombocytopenia and respiratory failure; in Case 2, increase in aminotransferase levels and respiratory failure) and in time according to the laboratory routine. The laboratory assessment flow chart is shown in Table 1.

Hantavirus infection is a worldwide disease that is often underdiagnosed due to its rapid progression and unfavorable evolution. It is caused by an arbovirus of the Bunyaviridae family, genus Hantavirus. The disease has two clinical presentations: hemorrhagic fever with renal syndrome (HFRS) and Hantavirus cardiopulmonary syndrome (HCPS). ${ }^{1}$ The HFRS is characterized by fever, chills, retroorbital headache, photophobia, myalgia, abdominal pain, nausea and vomiting, cranial exanthema, as well as petechiae in the soft palate and axillary region, which develop into hypotension and kidney failure. ${ }^{2}$ The HCPS presents as fever, diarrhea, headache, myalgia, nausea, vomiting, sudoresis and vertigo, with cardiovascular and respiratory involvement, similar to the adult respiratory distress syndrome. ${ }^{3}$ The differential diagnosis with other hemorrhagic fevers is mandatory for adequate clinical conduct, as well as environmental control. ${ }^{4}$ The differential diagnosis with severe community-acquired pneumonias is clinically and epidemiologically relevant. The anamnesis, which includes environmental 
Table 1. Laboratory assessment of Hantavirus infection cases

\begin{tabular}{lcccc}
\hline \multirow{2}{*}{ Laboratory tests } & \multicolumn{2}{c}{ Case $\mathbf{1}$} & \multicolumn{2}{c}{ Case 2 } \\
& Admission & ICU discharge & Admission & ICU discharge \\
\hline Creatinine (mg/dL) & 1.0 & 0.7 & 0.8 & 0.9 \\
\hline Urea (mg/dL) & 39.0 & - & 18.0 & 24.0 \\
\hline Hematocrit (\%) & 45.4 & 42.8 & 32.6 & 32.7 \\
\hline Hemoglobin (mg/dL) & 15.0 & 14.2 & 10.6 & 10.5 \\
\hline Leukocytes (mm $\left.{ }^{3}\right)$ & 6,320 & 4,700 & 2,600 & 6,920 \\
\hline Platelets (mm $\left.{ }^{3}\right)$ & 43,000 & 45,000 & 131,000 & 303,000 \\
\hline pH & 7.4 & 7.5 & 7.5 & 7.4 \\
\hline PaO2 (mmHg) & 67.1 & 81.0 & 64.6 & 104.9 \\
\hline HCO3 (mmol/L) & 15.7 & 23.6 & 22.6 & 18.6 \\
\hline Base excess (mEq/L) & -5.9 & +2.1 & +1.3 & -3.5 \\
\hline Oxygen arterial saturation (\%) & 94.2 & 96.8 & 94.3 & 98.0 \\
\hline Aspartate aminotransferase (UI/L) & 28.0 & 40.0 & 27.0 & 173.0 \\
\hline Alanine aminotransferase (UI/L) & 23.0 & 28.0 & 8.0 & 129.0 \\
\hline Lactate dehydrogenase (mg/dL) & 592.0 & - & 209.0 & 414.0 \\
\hline Serology for HIV* & - & Non-reactive & Non-reactive & - \\
\hline Serology for Leptospirosis*** & - & Non-reactive & - & Non-reactive \\
\hline Serology for Hantavirus*** & - & IgM Positive & - & IgM Positive \\
\hline
\end{tabular}

* ELISA, enzyme linked immunosorbent assay

** MAT, microscopic agglutination test - Laboratório Central do Estado, RS, Brazil

**: IgM antibody capture - Instituto Adolf Lutz, SP, Brazil

assessment, is crucial, especially in severe cases. However, the empiric and intensive support therapies are mandatory to decrease the morbidity and mortality. The confirmation of suspected diagnoses will be adequate only when the collection follows the incubation period of each infectious disease, taking into account the most prevalent ones in the community. A proactive attitude by the epidemiologic surveillance system regarding suspected cases of HCPS and its clinical investigation are crucial to consolidate the knowledge of the natural history of the disease.

[Braz J Infect Dis 2011;15(3):298-299]@Elsevier Editora Ltda.

\section{REFERENCES}

1. Butler JC, Peters CJ. Hantaviruses and hantavirus pulmonary syndrome. Clin Infect Dis. 1999; 19:387-95.

2. Schamljohn C, Hjelle B. Hantavirus: a global disease problem. Emerg Infect Dis. 1997; 3:95-104.

3. Figueiredo, LTM. Febres hemorrágicas por vírus no Brasil. Rev Soc Bras Med Trop. 2006; 39(2):203-10.

4. Chapman LE, Ellis BA, Koster FT et al. Discriminators between hantavirus-infected and uninfected persons enrolled in a trial of intravenous ribavirin for presumptive hantavirus pulmonary syndrome. Clin Infect Dis. 2002; 34:293-304 